\title{
Le français en Bulgarie dans le contexte de la politique culturelle de la France aux XIX et $\mathrm{XX}^{\mathrm{e}}$ siècles
}

\section{Raïa Zaïmova}

\section{(QpenEdition Journals}

Édition électronique

URL : https://journals.openedition.org/dhfles/286

DOI : $10.4000 /$ dhfles. 286

ISSN : 2221-4038

\section{Éditeur}

Société Internationale pour l'Histoire du Français Langue Étrangère ou Seconde

\section{Édition imprimée}

Date de publication : 1 janvier 2007

Pagination : 149-156

ISSN : 0992-7654

\section{Référence électronique}

Raïa Zaïmova, «Le français en Bulgarie dans le contexte de la politique culturelle de la France auX XIX et XXe siècles », Documents pour l'histoire du français langue étrangère ou seconde [En ligne], 38/39 | 2007, mis en ligne le 16 décembre 2010, consulté le 27 mai 2021. URL : http:// journals.openedition.org/dhfles/286 ; DOI : https://doi.org/10.4000/dhfles.286

Ce document a été généré automatiquement le 27 mai 2021 


\title{
Le français en Bulgarie dans le contexte de la politique culturelle de la France aux XIX ${ }^{\mathrm{e}}$ et $\mathrm{XX}^{\mathrm{e}}$ siècles
}

\author{
Raïa Zaïmova
}

1 Il n'y a pas longtemps que la France a commémoré le centenaire de la séparation de l'Église et de l'État de 1905 - date très importante dans l'histoire moderne et résultat de plusieurs décennies de luttes anti-cléricales et de combat de l'esprit des Lumières. En même temps, c'est un moment crucial pour la laïcité - notion qui repose sur des principes humanistes forgés au cours de l'histoire - et pour le progrès des sciences qui doivent être libérées de toute influence des groupes de pression religieux. L'intérêt général et le respect de l'individu doivent être les seuls cadres de ce progrès.

Dans cet ordre d'idées, il faut rappeler un phénomène important. Par la loi française de 1901 concernant le statut des congrégations catholiques en France, un grand nombre de leurs écoles sont fermées ${ }^{1}$. Mais celles qui se trouvent à l'étranger continuent leurs activités sans aucun problème de la part du gouvernement français et spécialement du ministère des Affaires étrangères. De même, les longues discussions qui mènent à la séparation de l'Église et de l'État en 1905, ne laissent aucune empreinte sur les congrégations à l'étranger, y compris en Bulgarie déjà État moderne (à partir de 1878) et indépendant (depuis 1908).

\section{Le français dans la Bulgarie ottomane}

3 Jusqu'à cette époque, la langue et la culture françaises ont été enseignées d'abord dans les collèges catholiques et en partie dans les écoles bulgares qui fonctionnaient sur le territoire ottoman dès les premières décennies du XIX ${ }^{e}$ siècle. Cette tradition a continué dans le cadre de l'État bulgare moderne - et ceci grâce aux capitulations signées entre la France et la Bulgarie pendant les années $1880^{2}$. J'aimerais mettre l'accent sur le fait que pendant les siècles précédents, conformément aux capitulations conclues pour la première fois entre François ${ }^{\text {er }}$ et Suleyman le Magnifique (1535/6), la France avait 
ouvert un grand nombre de consulats dans la Méditerranée orientale. Les Provençaux, sujets du roi et tous les Occidentaux qui n'avaient pas de tels rapports avec Constantinople profitaient de ce droit international pour naviguer et faire le commerce dans les provinces ottomanes. Ainsi, au début du XVIII ${ }^{\mathrm{e}}$ siècle, les consulats et les viceconsulats de France étaient au nombre de 48, ce nombre dépassant celui des Anglais et des autres sujets étrangers. Ce grand réseau, créé surtout au temps de Colbert, a bien fonctionné jusqu'à la Révolution de 1789. Entre temps, dès l'époque de l'Ancien Régime, la politique culturelle de la France dans l'Empire ottoman fait ses premiers pas dans l'européanisation du pays ${ }^{3}$. Dans ce sens, l'éducation des sujets du sultan même avant la guerre de Crimée (1854-1855) occupe une place prépondérante dans les programmes ministériels français. La réalisation de ceux-ci est encouragée par les réformes (le Tanzimat) dans l'empire du sultan. La France avait abandonné depuis longtemps ses projets de Croisade et de monarchie universelle et tiré un profit culturel des besoins intérieurs de la Turquie en matière de formation d'experts pour l'armée et la haute administration. Elle soutient largement l'intégrité de l'Empire ottoman. Les collèges des lazaristes, l'école de médecine, et puis le lycée impérial de Galatasaray accueillent des élèves de toutes les nationalités - chrétiens et musulmans, sujets du sultan, ainsi que des Levantins. Lors de l'ouverture du lycée laïc en 1868, le directeur français déclare qu'il s'agit de « développer et consolider l'influence française en Orient en lui donnant pour base une propagande intellectuelle, servir les intérêts de la France en raffermissant par la diffusion des connaissances [...] cet empire si chancelant et ébranlé, dont l'intégrité est une condition première dans l'équilibre européen. ${ }^{4}$.

4 En ce qui concerne les Bulgares qui ont fait leurs études à l'école de médecine et au lycée impérial de Galatasaray, le directeur les considère comme ses «meilleurs élèves, dociles, laborieux, et les autres les moins bons, paresseux, indisciplinés et immoraux $»^{5}$. Parmi eux, on pourrait citer les noms des médecins Christo Stambolski, J. P. Liubenov, A. P. Granitzki, P. S. Bobekov, S. S. Bobtchev ${ }^{6}$. Constantin Vélitchkov (1855-1907) écrivain, traducteur, peintre, homme politique - auteur de pièces de théâtre, dont les premières représentations ont eu lieu à Galatasaray, a aussi terminé ses études au lycée (1874). Les noms et le nombre des Bulgares de cet établissement ne sont pas tout à fait identifiés dans les archives. Il en est de même pour les élèves des autres nations balkaniques.

5 À partir de la seconde moitié du XIX siècle, la France réussit à former au Levant un système culturel et éducatif grâce aux congrégations catholiques. Ce système qui a fonctionné jusque dans les années $50 \mathrm{du} \mathrm{XX}^{\mathrm{e}}$ siècle rappelle dans un certain sens celui des consulats, mentionné ci-dessus. Ceci relève de l'évolution des intérêts français dans le Bassin méditerranéen et $\mathrm{du}$ désir de propager le progrès scientifique et civilisationnel des Lumières dans ce coin du monde.

6 À part les écoles des lazaristes à Constantinople, Salonique et Smyrne qui demeurent toujours sur le territoire de la Turquie, un certain nombre de congrégations catholiques s'installent sur le sol balkanique pendant les années 60-70 du XIX ${ }^{\mathrm{e}}$ siècle. Il s'agit des augustins de l'Assomption à Plovdiv et à Andrinople, des sœurs de Saint Joseph de l'Apparition, des sœurs oblates et celles de Notre Dame de Sion qui établissent leurs écoles pendant les années 1880 à Sofia, Varna, Plovdiv, Roussé, Bourgas, Yambol 7 . Selon un diplomate français contemporain, le collège des augustins à Plovdiv, ouvert en 1863, donc « 15 ans avant la délivrance de la Bulgarie », était très renommé pour la formation 
des jeunes: «c'est parmi les anciens élèves que la jeune Bulgarie avait recruté ses premiers fonctionnaires $»^{8}$.

\section{Le français dans la Bulgarie indépendante}

7 Un autre groupe d'institutions culturelles: l'Alliance française, qui ouvre ses portes en 1904 à Sofia et après, dans une dizaine de villes de province, et l'Institut français ouvert en 1923 à Sofia ${ }^{9}$, sont subventionnées et dirigées, de pair avec les congrégations catholiques, par le service spécial des Affaires étrangères de France, appelé Les Euvres. Constitué pendant les années $80 \mathrm{du} \mathrm{XIX}^{\mathrm{e}}$ siècle, ce service a pour objectif la propagation de la langue et de la culture françaises à l'étranger. Au début du XX siècle, lorsque les Affaires étrangères n'ont pas encore un fonctionnaire spécial (nommé "attaché culturel »), les efforts de la diplomatie française sont orientés vers la diffusion de la langue comme langue des communications internationales. Ces efforts augmentent d'autant plus lorsque les États-Unis font des démarches pour imposer l'anglais comme langue internationale. En fait, l'anglais s'impose dans les documents à caractère politique et international à partir de 1919, lorsque le traité de Versailles est signé également en anglais ${ }^{10}$. En effet, les congrégations catholiques jouent un rôle primordial dans la propagation de la langue et de la culture françaises à l'étranger. Malgré la séparation de l'Église et de l'État, leurs actions hors de France sont énormes et toujours placées sous l'égide du ministère des Affaires étrangères. Certaines activités, surtout pendant les périodes de guerre, sont subventionnées également par le Vatican.

8 En 1900 le nombre des personnels des Euvres à l'étranger s'élève à 50000 . Ces institutions sont les agents traditionnels de la langue et de la culture françaises et jouissent d'une grande autorité, malgré les dispositions républicaines en France. En 1902, l'aide attribuée aux congrégations représente $93 \%$ du total du chapitre des Euvres. La statistique montre également que le Maroc et les pays d'Orient ont reçu une certaine aide financière pour la période qui précède la Première Guerre mondiale. D'autre part, grâce à une autre subvention du Vatican, la France réussit à écarter la concurrence des congrégations catholiques d'Autriche-Hongrie. Ce phénomène devient évident, surtout en Bulgarie, après la Première Guerre mondiale, lorsque l'ancien État Autriche-Hongrie n'existe plus. De cette manière les écoles catholiques en Bulgarie demeurent exclusivement françaises. Leur personnel enseignant comprend non seulement des citoyens de France, mais aussi des Autrichiens catholiques et des Bulgares laïss de confession orthodoxe et de rite catholique ${ }^{11}$. Le recrutement se fait comme à l'époque de l'Empire ottoman: chaque enfant, nonobstant sa religion, a le droit de s'y inscrire. Le catholicisme n'est pas imposé, et toujours comme à l'époque ottomane, les élèves travaillent dans une ambiance de tolérance religieuse. On accueille des Bulgares orthodoxes et catholiques, des juifs, des Arméniens, à côté des enfants du personnel des missions diplomatiques et commerciales dans le pays. Par exemple, en 1917, le nombre des élèves orthodoxes de Saint-Joseph de l'Apparition à Sofia est de 280 , les catholiques étrangères sont 128 , et 192 sont des juives. La convention culturelle franco-bulgare de 1936 précise et légalise les activités françaises et catholiques en Bulgarie. Sur ce point, une collaboration étroite existe entre les deux pays. Les examens de maturité sont présidés par le ministre de France à Sofia. Les diplômes délivrés sont reconnus par un grand nombre de pays et donnent droit d'accès aux universités de 
l'Europe ${ }^{12}$ - un phénomène qu'on cherche actuellement à rétablir en Bulgarie après 50 ans de régime communiste. De même, pendant la Seconde Guerre mondiale, le gouvernement de Vichy trouve un biais pour soutenir les activités éducatives et culturelles dans le cadre des CEuvres $^{13}$.

Malgré l'état de guerre, des intellectuels bulgares - des professeurs universitaires, des écrivains, médecins, architectes, juristes, peintres - reçoivent régulièrement leurs abonnements à la presse périodique française. Parmi eux on trouve Nikola Mavrodinov (historien de l'art et archéologue), Bogdan Filov (archéologue, premier ministre de 1940 à 1943), Elissavéta Bagriana (poète), Petko Stajnov (juriste), Anna Kamenova (écrivain), Nikolaj Liliev (poète et dramaturge), Fany Popova-Moutafova (écrivain), etc. Les salles de l'Alliance continuent à accueillir un grand public intéressé par l'histoire littéraire et la civilisation françaises. Les livres pour les bibliothèques de l'Alliance et de l'Institut sont fournis via la Turquie en évitant les zones occupées. En 1944, vers la fin de la Seconde Guerre mondiale, les écoles françaises en Bulgarie (y compris celles de Skopié et Bitolia qui faisaient partie des territoires annexés) ont plus de 5000 élèves. À elle seule la petite bibliothèque de l'Alliance à Sofia (il y en avait deux) comprend 16500 livres et périodiques. Pendant les années 1950, elle est stockée avec celle de l'Institut dans les locaux de l'ambassade de France et, après 1957, transportée en grande partie à l'université de Sofia ${ }^{14}$.

\section{Le français dans la Bulgarie communiste et post- communiste}

10 Après la guerre, le nouveau régime communiste procède à la fermeture définitive des collèges. Au niveau diplomatique, la Bulgarie déclare qu'elle compte demander des instituteurs laïcs parce que la France républicaine n'a pas le droit d'imposer une éducation religieuse dans un pays étranger. Mais en avril 1947, l'opposition bulgare en la personne de l'agrarien Nikola Petkov résume ainsi sa position devant l'Assemblée nationale: «Le peuple bulgare tire profit de la culture française, de la clarté et des idées de fraternité, égalité et liberté que la France a répandues dans ce pays ». Par contre, le ministre communiste Vassil Kolarov déclare que les «écoles françaises en Bulgarie sont un héritage du temps des capitulations, elles constituent une anomalie qui ne saurait être tolérée dans un État souverain moderne $»^{15}$. Les arguments de la diplomatie française ne sont pas pris en considération dans les longs pourparlers franco-bulgares, et la décision des autorités bulgares est définitive: par décret de l'Assemblée générale, publié dans le journal d'État (3 août 1948), les collèges français sont fermés pour toujours.

11 Entre temps, par une ordonnance du 13 avril 1945, le service des CEuvres est transformé en Direction générale des relations culturelles et des œuvres françaises à l'étranger. L'argument de Charles de Gaulle est que c'est «par de libres rapports intellectuels et moraux, établis entre nous-mêmes et les autres que notre influence culturelle peut s'étendre à l'avantage de tous et qu'inversement peut s'accroître ce que nous valons ${ }^{16}$. La France regagne son influence intellectuelle en Bulgarie. La longue tradition peut renaître à petits pas chancelants dans un autre système d'éducation et de diffusion de sa culture. 

la Bulgarie est devenue membre de l'Organisation internationale de la Francophonie, puis de l'Agence universitaire de la Francophonie. L'édition LIBUL, qui est un inventaire bibliographique de la littérature bulgare d'expression française, paru en 2004, comprend 3805 titres de livres et 2546 titres d'articles de périodiques publiés par des Bulgares en français entre 1842 et $1995^{17}$. Ce grand nombre donne une idée de la place $\mathrm{du}$ français langue étrangère dans l'espace linguistique national et ouvre l'horizon à une future recherche de type comparatiste.

En guise de conclusion, je mettrai l'accent sur le fait que les collèges catholiques en Bulgarie occupaient une place importante dans la politique culturelle de la France jusqu'aux années 50 du siècle dernier. La séparation de l'Église et de l'État en 1905 n'a pas empêché les activités des Euvres, au contraire, l'enseignement et la propagation de la langue des Lumières, soutenue par l'État français républicain au cours des années, a maintenu la vieille tradition francophone et balkanique. Cette politique flexible, due aux capitulations, a été rompue à la fin des années 40 par les gouvernements bulgares qui se disaient pourtant « républicains » et « laïcs ».

\section{NOTES}

1. Archives nationales, F17 Instruction publique, dossiers 12479 à 12504, 12496.

2. Mitev, Pl. (1996), Dărzavnata reglamentatzija na gradskoto stopanstvo v bălgarskite zemi prez XVIII v. [Le règlement étatique de l'économie en ville dans les terres bulgares au XVIII ${ }^{\mathrm{e}} \mathrm{s}$ ], Săzdavane i razvitie na moderni instoutzii v bălgarskoto văzroznesko obchtestvo (Sofia), p. 53 sq.

3. Zaïmova, R. (2007), Voyager vers l'« autre " Europe (images françaises des Balkans ottomans, XVI ${ }^{e}$ XVIII ${ }^{e}$ siècles), Istanbul : éd. Isis.

4. Archives nationales, $\mathrm{F}^{17}$ Instruction publique, dossier 4613.

5. Ibidem.

6. Gentchev, N. (1979), Frantzija v bulgarskoto douhovno Vazrazdane [La France dans l'Éveil spirituel bulgare], Sofia, p. 251 sq.

7. Archives du ministère des Affaires étrangères, Relations culturelles, 1945-1959, sous-série 1945-1947, dossier 188 (Bulgarie).

8. Ibidem, Dossier 63.

9. Kolev, N. (1994), « Les premières péripéties de la formation de la chaire de philologie romane à l'Université à Sofia ", $70^{e}$ anniversaire du département d'études romanes, Colloque international, Sofia, décembre 1993, Sofia : Presse universitaire, pp. 103-110.

10. Pitti Ferrandi, Fr. (2001), «Le français, langue diplomatique », La francophonie à travers «la Revue de l'AMOPA » (Paris), pp. 12-15.

11. Pigniau, Fr. (1995), Histoire de la diplomatie culturelle des origines à 1995. Paris: La Documentation française, pp. 21-35.

12. Archives du ministère des Affaires étrangères, Relations culturelles 1945-1959, sous-série 1945-1947, dossier 188 (Bulgarie).

13. Archives du ministère des Affaires étrangères, Guerre 1939-1945, Vichy-๕Euvres, dossier 32b (Bulgarie).

Documents pour l'histoire du français langue étrangère ou seconde, 38/39 | 2007 
14. Archives du ministère des Affaires étrangères, Relations culturelles, 1945-1959, sous-série 1945-1947, dossier 188 (Bulgarie) ; sous-série Enseignement 1948-1959, dossier 62 (Bulgarie).

15. Archives du ministère des Affaires étrangères, Europe 1944-1960, dossier 113 (Bulgarie).

16. Pigniau, Fr., op. cit., p. 69.

17. LIBUL. Inventaire bibliographique de la littérature bulgare d'expression française. БИБЛИОГРАФСКИ УКАЗАТЕЛ НА ФРЕНСКОЕЗИЧНАТА ЛИТЕРАТУРА ОТ БЪЛГАРСКИ АВТОРИ. 1842-1995. Sofia, 2004. 402 p. (co-auteursS. Boteva, I. Guécheva, L. Guénova, D. Ignatova, I. Iliev, R. Stantchéva, J. Tafradjiiska, réd. St. Atanassov, Al. Vuillemin).

\section{RÉSUMÉS}

Les collèges catholiques en Bulgarie occupaient une place importante dans la politique culturelle de la France jusqu'aux années 1950. La séparation de l'Église et de l'État en 1905 n'avait pas empêché les activités des Euvres dans ce pays balkanique où la tradition francophone était maintenue et le Quai d'Orsay continuait de collaborer avec les autorités bulgares. En profitant parfois des subventions du Vatican, la France avait réussi à écarter la concurrence des congrégations autrichiennes. Les élèves - orthodoxes et catholiques, Arméniens et juifs travaillaient dans une ambiance de tolérance religieuse. Cette politique flexible, due aux capitulations depuis l'époque ottomane, a été rompue à la fin des années 1940 par les gouvernements communistes qui se disaient « républicains » et « laïcs ».

Until the 1950s the Catholic colleges in Bulgaria were an important element of France's cultural policies in the Balkans. The 1905 separation of Church from State had not prevented the Cuvres from deploying their activity in the Balkan countries to maintain the Francophone tradition. To that effect the French Foreign Ministry collaborated closely with the Bulgarian authorities. Sometimes France availed herself of the opportunity, offered by the subsidies coming from the Vatican, for overcoming the competition of the Austrian congregations. Pupils, no matter whether Orthodox or Catholic, Armenians or Jews, worked in an atmosphere of religious tolerance. Toward the end of the 1940s the Communist government, claiming to be « republican » and « lay», put an abrupt end to these flexible policies.

\section{INDEX}

Keywords : French language, cultural policy, Bulgaria, religious tolerance, communism

Mots-clés : langue française, politique culturelle, Bulgarie, tolérance religieuse, communisme

\section{AUTEUR}

\section{RAÏA ZAÏMOVA}

Institut des études balkaniques, Sofia, Bulgarie 